\title{
Fabricación y caracterización de membranas cerámicas tubulares para microfiltración
}

\author{
J. MARCHESE ${ }^{1}$, C. ALMANDOZ ${ }^{1}$, M. AMARAL ${ }^{1}$, L. PALACIO, J. I. CALVO, P. PRADANOS y A. HERNANDEZ \\ (1)Laboratorio de Ciencia de Superficies y Medios Porosos (UNSL-CREACYT), 5700 San Luis, Argentina. \\ Dpto. Termodinámica y Física Aplicada, Facultad de Ciencias, Universidad de Valladolid, 47071 Valladolid, España
}

\begin{abstract}
En este trabajo se describe el método de preparación y la caracterización de diversas membranas cerámicas tubulares con utilidad en el campo de la microfiltración. Se trata de membranas compuestas formadas por un soporte macroporoso que le confiere resistencia mecánica y una capa delgada microporosa o superficie activa depositada sobre el soporte que es la que determina las características retentivas de la membrana. Se prepararon diversas versiones de estas membranas a partir de materias primas cerámicas controlando, entre otras, variables como: el tamaño de partículas, la formulación química de la pasta y su tratamiento térmico. El material del soporte y de la capa activa se obtiene a partir de barbotinas a base de caolín, arcilla, feldespato, alúmina, etc. La morfología estructural del soporte, capa activa y membrana compuesta se ha estudiado mediante técnicas de AFM (Microscopía de Fuerza Atómica) y permeabilidades hidráulicas. Las propiedades estructurales y permeoselectivas de estas membranas cerámicas, hacen que sean apropiadas para procesos de microfiltración.
\end{abstract}

Palabras Clave: Materiales porosos, membranas, sintesis de membranas tubulares, microfiltración, microcopía de fuerza atómica.

Fabrication and characterization of microfiltration tubular ceramic membranes

A method to prepare and characterize different microfiltration ceramic membranes is described here. The membranes are composite and consist in a macroporous support, giving high mechanical resistance, onto which a thin microporous layer is deposited. This active film determines the retention properties of the membrane. Several versions of these membranes were prepared by controlling: particle size, chemical formulation of the paste and its thermal treatment. Both the support and the active layer were obtained from slips consisting in caolin, clay, feldespatum etc. The morphology of the active layer, support and composite membrane have been investigated by AFM (Atomic Force Microscopy) and hydraulic permeabilities. Structural properties and permselectivity make these membranes appropriate to be efficiently used in microfiltration processes..

Keywords: Porous materials, membranes, synthesis of tubular membranes, microfiltration, atomic force microscopy.

\section{INTRODUCCIÓN}

Los factores más determinantes en la viabilidad de un proceso de separación por membranas son el grado de separación conseguido en el proceso y el tiempo de vida de la membrana utilizada. Para la mayoría de los diseños de procesos de separación por membranas, sobre todo aquellos en que la fuerza impulsora es un gradiente de presión, existe gran número de membranas comerciales, poliméricas o cerámicas, que permiten conseguir factores de recuperación similares o superiores a los convencionales (1). No obstante, en muchos casos el tiempo de vida de estas membranas puede hacer que estos procesos no sean viables desde el punto de vista económico. Las membranas cerámicas han contribuido en gran medida a salvar este problema. Sobre todo debido a una serie de factores como son, la mayor permeabilidad que se traduce en que con este tipo de membranas el área de filtración requerida sea significativamente menor; y la alta resistencia térmica, química y mecánica que permiten una recuperación casi total de este tipo de filtros $(2,3)$.

Independientemente del tipo de módulo con el que se esté trabajando (configuración externa de la membrana), la configuración interna de las membranas cerámicas es similar a las poliméricas; es decir, están formadas por dos o más capas. Éstas consisten en una o varias capas de soporte poroso, que suelen estar fabricadas de materiales baratos y con procesos convencionales y una capa selectiva mucho más elaborada. Dado que la estructura de la membrana va ha determinar las características de operatividad de esta, el proceso de fabricación y los materiales empleados en su fabricación son de gran importancia (4).

En este trabajo se propone un método de síntesis de membranas cerámicas tubulares asimétricas de microfiltración, usando un soporte común a todas ellas. Para determinar la influencia del soporte sobre la capa activa, se han fabricado y analizado tanto las membrana asimetricas como tal junto con membranas de geometría externa similar pero usando solamente la pasta de la capa activa. La caracterización se ha realizado utilizando medidas de permeabilidad hidráulica que permiten determinar el diámetro hidráulico equivalente y microscopía de fuerza atómica (AFM). (5)

\section{MATERIALES Y METODOS EXPERIMENTALES}

\subsection{Síntesis}

Como se ha mencionado anteriormente se han preparado cuatro tipos de membranas tubulares cuya diferencia principal es su tamaño de poro. No obstante, con el fin de determinar la 


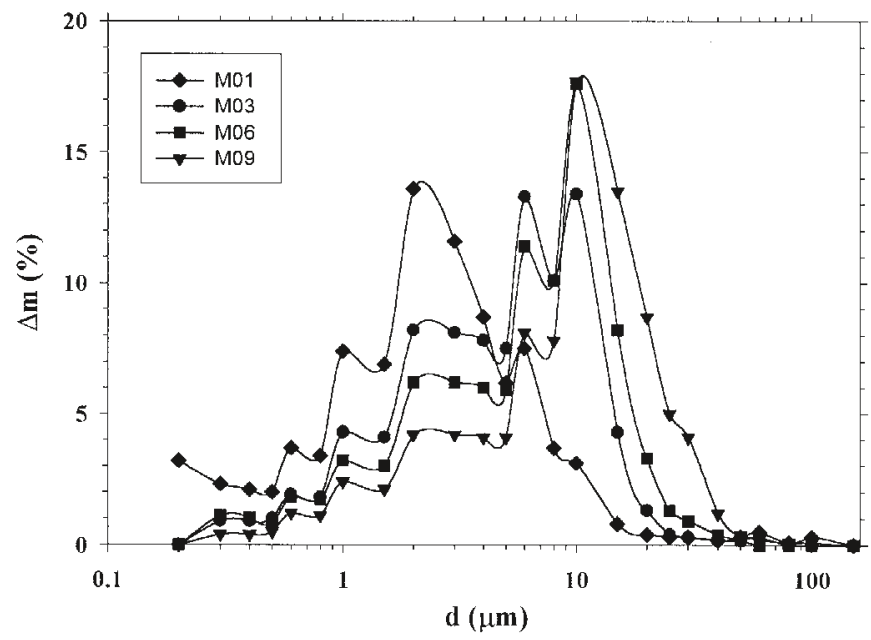

Fig. 1.- Distribuciones de tamaños de partículas obtenidas de la pasta cerámica para todas las membranas analizadas.

influencia de la adhesión de la capa activa al soporte poroso, se han caracterizado tanto las membranas soportadas como las fabricadas exclusivamente con la pasta empleada para la capa activa. En la Tabla I se da la nomenclatura que identifica cada una de las muestras con las que se ha trabajado. Las muestras de membrana obtenidas consisten en tubos de $10 \mathrm{~mm}$ de diámetro interno y $12 \mathrm{~mm}$ de diámetro externo.

\subsubsection{SOPORTE MACROPOROSO}

El soporte macroporoso S25 fue preparado por el método de extrusión. Se utilizó una extrusora con boquilla de geometría anular, con diámetro externo de $12 \mathrm{~mm}$ e interno de $10 \mathrm{~mm}$. Las materias primas utilizadas en el soporte fueron: alúmina, arcilla, cuarzo, carbonato cálcico, feldespato, bentonita y talco en las proporciones adecuadas, junto con polietilenglicol y glicerina como aditivos orgánicos. Se realizó un análisis granulométrico por rayos-X (Sedigraph de Micromeritis), cuya dis-

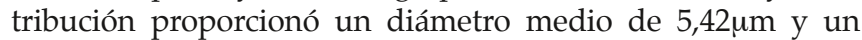
diámetro modal de $53,94 \mu \mathrm{m}$. El valor de la densidad de las partículas de acuerdo con la bibliografía es de $2,6 \mathrm{~g} / \mathrm{cm}^{3}$. Las partículas se suspenden en agua, con el fin de lograr la completa disgregación de todos los componentes, hasta lograr una disolución homogénea. Posteriormente, la pasta se seca extrayendo el agua sobre placas de yeso. Cuando su humedad es del orden del $20 \%$ la pasta se introduce en la extrusora para la fabricación de los tubos. Los tubos obtenidos se secan a temperatura ambiente (1 día aproximadamente), luego se secan en estufa a $100{ }^{\circ} \mathrm{C}$ durante otro día y finalmente sufren un proceso de recocido a temperaturas linealmente crecientes hasta unos $1100{ }^{\circ} \mathrm{C}$ al cabo de 24 horas (6).

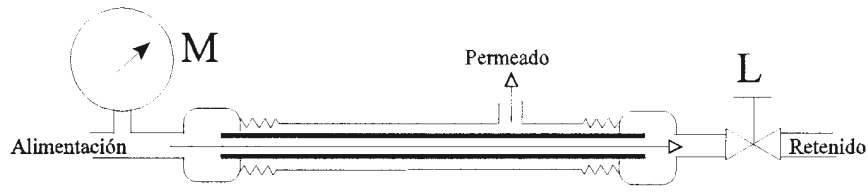

Fig. 2.- Célula de membrana de geometría tubular utilizada para la determinación de la permeabilidad hidraúlica.
TABla I: DESCRIPCIÓN DE LAS MEMBRANAS UTILIZADAS. LAS MEMBRANAS MS POSEEN COMO CAPA ACTIVA EL MISMO MATERIAL QUE LAS M PERO ÉSTA CONSISTE EN UNA CAPA MUY FINA SOBRE EL SOPORTE S25.

\begin{tabular}{|c|c|c|}
\hline Membrana & Descripción & Tamaño de poro Nominal \\
\hline S25 & Soporte simétrico & $2,5 \mathrm{~mm}$ \\
M01 & Membrana Simétrica & $0,1 \mathrm{~mm}$ \\
M03 & Membrana Simétrica & $0,3 \mathrm{~mm}$ \\
M06 & Membrana Simétrica & $0,6 \mathrm{~mm}$ \\
M09 & Membrana Simétrica & $0,9 \mathrm{~mm}$ \\
MS01 & Membrana Asimétrica & $0,1 \mathrm{~mm}$ \\
MS03 & Membrana Asimétrica & $0,3 \mathrm{~mm}$ \\
MS06 & Membrana Asimétrica & $0,6 \mathrm{~mm}$ \\
MS09 & Membrana Asimétrica & $0,9 \mathrm{~mm}$ \\
\hline
\end{tabular}

\subsubsection{CAPA ACTIVA}

Con la misma configuración tubular que el soporte, y utilizando el método de colada sobre moldes tubulares de yeso, se han preparado 4 membranas de estructura simétricas que hemos denominado M01, M03, M06 y M09. La formulación de la pasta es común para todas, y consiste de una mezcla de alúmina, cuarzo y arcilla, añadiendo ftalatos alquílicos como aditivos orgánicos. La densidad de las partículas para esta pasta es de $2,7 \mathrm{~g} / \mathrm{cm}^{3}$ de acuerdo con los datos de la bibliografía. La diferencia entre las membranas se debe a las distintas distribuciones de tamaño de las partículas, las cuales se consiguen con el diferente tiempo de molienda. La molienda de las pastas se realizó en un molino de bolas Restch. Lo tiempos de molienda así como los valores característicos de la distribución se presentan en la Tabla II para las cuatro membranas. En la Figura 1 se presentan las distribuciones de partículas obtenidas de la pasta utilizada para todas las membranas. Antes de la colada, las pastas se ajustan a un peso específico de 1,2 g/ $\mathrm{cm}^{3}$ y una viscosidad de $60 \mathrm{mPa} \cdot \mathrm{s}$. De la misma forma que para el soporte, las membranas se secan primero a temperatura ambiente y posteriormente a $100^{\circ} \mathrm{C}$. Finalmente se sinteriza a $1200^{\circ} \mathrm{C}$ en dos etapas, primero hasta $500^{\circ} \mathrm{C}$ a una velocidad de calentamiento de $5^{\circ} \mathrm{C} /$ minuto y posteriormente a $10^{\circ} \mathrm{C} /$ minuto hasta los $1200^{\circ} \mathrm{C}(6)$.

\subsubsection{MEMBRANA COMPUESTA}

Para las membranas compuestas (MS01, MS03, MS06 y MS09) el soporte, preparado como se ha mencionado en el apartado 2.1.1, se utiliza como molde para la colada de las pastas utilizadas en el apartado 2.1.2. En este caso la diferencia es que en el proceso de colada, la capa activa depositada es de unas decenas de micra frente a $1 \mathrm{~mm}$ para las membranas simétricas. Las membranas así formadas, siguen un proceso de secado y sinterizado idéntico al caso anterior (4).

\subsection{Medida del Flujo y la Permeabilidad Hidráulica}

Con el fin de determinar el radio hidráulico, se realizaron 


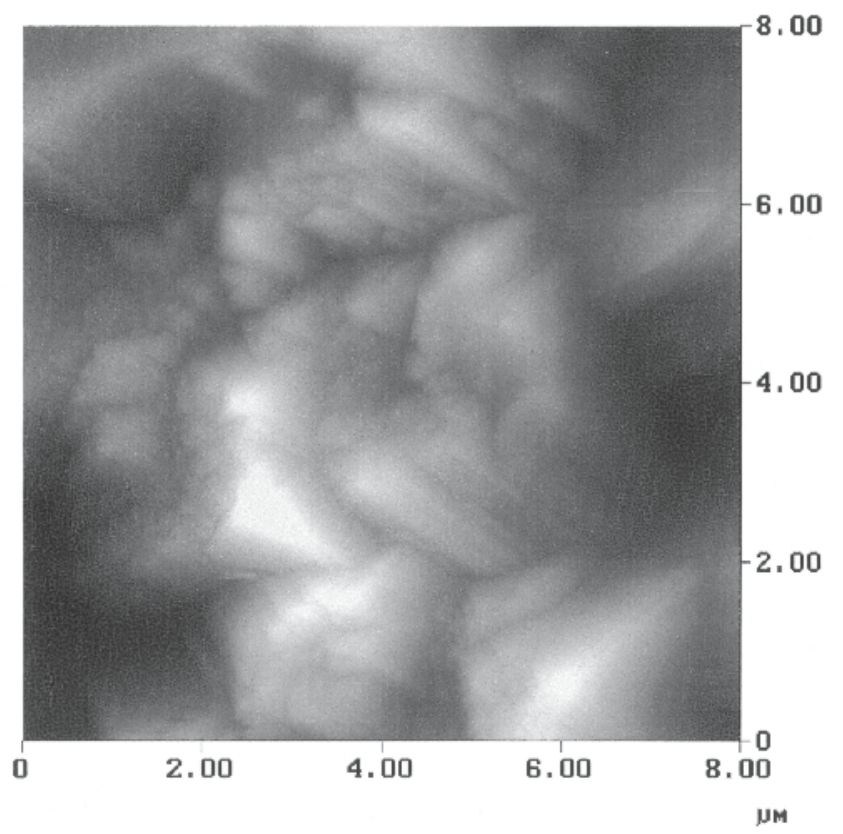

Fig. 3.- Imagen topográfica de la superficie de la membrana M01, obtenida por AFM.

medidas de flujo de agua con las membranas tubulares anteriormente descritas; con dimensiones: longitud 0,2 m, diámetro interno $0,01 \mathrm{~m}$ y espesor de la pared $0.001 \mathrm{~m}$. Estas experiencias se realizaron en una célula para membranas de geometría tubular como la representada en la Figura 2. El sistema se alimenta con agua destilada y desionizada utilizando una bomba peristáltica. La presión del sistema se regula mediante una válvula situada a la salida de la célula (L) y se mide con un manómetro a la entrada de ésta (M). Para estos experimentos, el flujo de recirculación de retenido fue de $0,25 \mathrm{~L} / \mathrm{s}$ y la diferencia de presión aplicada a través de la membrana $(\Delta \mathrm{p})$ estuvo entre 104 y $105 \mathrm{~Pa}$. Debido a que la longitud de la membrana es pequeña y no se utilizan grandes flujos de recirculación de retenido, se puede suponer que la caída de presión a lo largo de la membrana es despreciable. El flujo volumétrico para un determinado valor de $\Delta \mathrm{p}$, se determina mediante volumetría cinética.

\subsection{Microscopía de Fuerza Atómica}

Las imágenes de Microscopía de Fuerza Atómica (AFM) se han realizado con un Nanoscope IIIA de Digital. Éstas se han obtenido utilizando las técnicas: de contacto intermitente con

TABlA II: TIEMPO DE MOLIENDA Y PARÁMETROS DE LAS DISTRIBUCIONES DE PARTÍCULAS OBTENIDAS PARA CADA UNA DE LAS MEMBRANAS SIMÉTRICAS.

\begin{tabular}{|c|c|c|c|}
\hline Membrana & $\begin{array}{c}\text { Tiempo de } \\
\text { Molienda }\end{array}$ & $\begin{array}{c}\text { Diámetro } \\
\text { Medio }(\mu \mathrm{m})\end{array}$ & $\begin{array}{c}\text { Diámetro } \\
\text { modal }(\mu \mathrm{m})\end{array}$ \\
\hline M01 & 48 horas & 2,4 & 3,79 \\
M03 & 10 horas & 5,14 & 7,6 \\
M06 & 180 minutos & 6,48 & 10,27 \\
M09 & 120 minutos & 10,23 & 16 \\
\hline
\end{tabular}
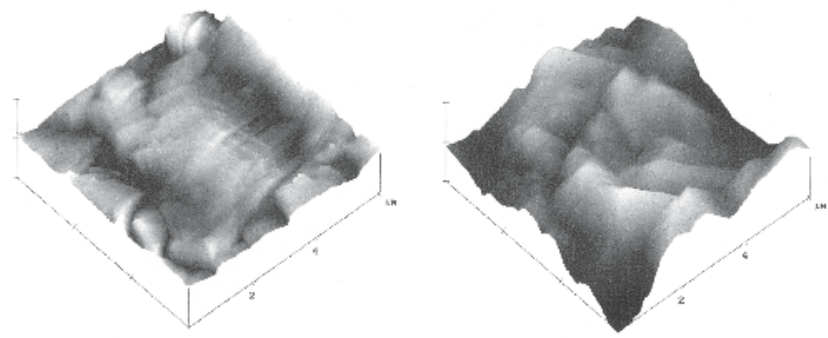

Fig. 4.- Imágenes tridimensionales de topografía AFM para las membranas M01 y MS01. Ambas imágenes presentan un rango en alturas de $2 \mu \mathrm{m}$.

una punta de brazo (cantilever) oscilante a su frecuancia natural de resonancia ("Tapping Mode") y de contacto. En el primer caso, se utiliza un solo brazo con punta de silicio, mientras que en el segundo, el brazo es doble con forma de $\mathrm{V}$ en cuyo extremo existe una punta de nitruro de silicio.

\section{RESULTADOS}

\subsection{Permebilidades y Radio Hidráulico}

En la Tabla III se dan los valores de permeabilidad hidráulica para cada una de las membranas que se han estudiado. De estos datos se puede resaltar que el soporte, como era de esperar, posee una permeabilidad muy superior a cualquiera de las otras membranas. En segundo lugar, las permeabilidades de las membranas soportadas son siempre más altas que las correspondientes simétricas con el mismo tamaño de poro. Esto era previsible, ya que en las membranas soportadas disminuye el espesor de la capa selectiva que es la que mayor resistencia opone al flujo.

La determinación del radio hidráulico se ha realizado a partir de la ecuación de Hagen-Poiseuille, que proporciona la permeabilidad, expresada en función de la porosidad, como:

$$
\mathrm{L}_{\mathrm{p}}=\frac{\mathrm{J}_{\mathrm{V}}}{\Delta \mathrm{p}}=\frac{\varepsilon \mathrm{r}_{\mathrm{h}}{ }^{2}}{8 \Delta \mathrm{x} \eta}
$$

donde Lp es la permeabilidad hidráulica, $\mathrm{J}_{\mathrm{V}}$ es el flujo en volumen de permeado, $\Delta$ p es la caída de presión a través de la

TABLA III: PERMEABILIDADES, POROSIDADES Y RADIOS HIDRÁULICOS PARA EL SOPORTE Y LAS MEMBRANAS SIMÉTRICAS Y COMPUESTAS.

\begin{tabular}{|c|c|c|c|}
\hline Membrana & $\begin{array}{c}\text { Permeabilidad } \\
\text { Hidráulica } \\
\text { Lp (m/s·Pa) }\end{array}$ & $\begin{array}{c}\text { Porosidad } \\
\mathbf{( \% )}\end{array}$ & $\begin{array}{c}\text { Radio } \\
\text { Hidráulico, } \\
\mathbf{r}_{\mathbf{h}}(\boldsymbol{\mu m})\end{array}$ \\
\hline S25 & $7,50 \cdot 10-7$ & 44,79 & 3,46 \\
M01 & $1,53 \cdot 10-9$ & 48,62 & 0,15 \\
M03 & $7,90 \cdot 10-9$ & 62,76 & 0,30 \\
M06 & $2,11 \cdot 10-8$ & 35,70 & 0,65 \\
M09 & $4,36 \cdot 10-8$ & 36,04 & 0,93 \\
MS01 & $1,35 \cdot 10-8$ & -- & -- \\
MS03 & $1,44 \cdot 10-8$ & --- & --- \\
M506 & $1,58 \cdot 10-8$ & --- & --- \\
MS09 & $8,69 \cdot 10-8$ & --- & --- \\
\hline
\end{tabular}




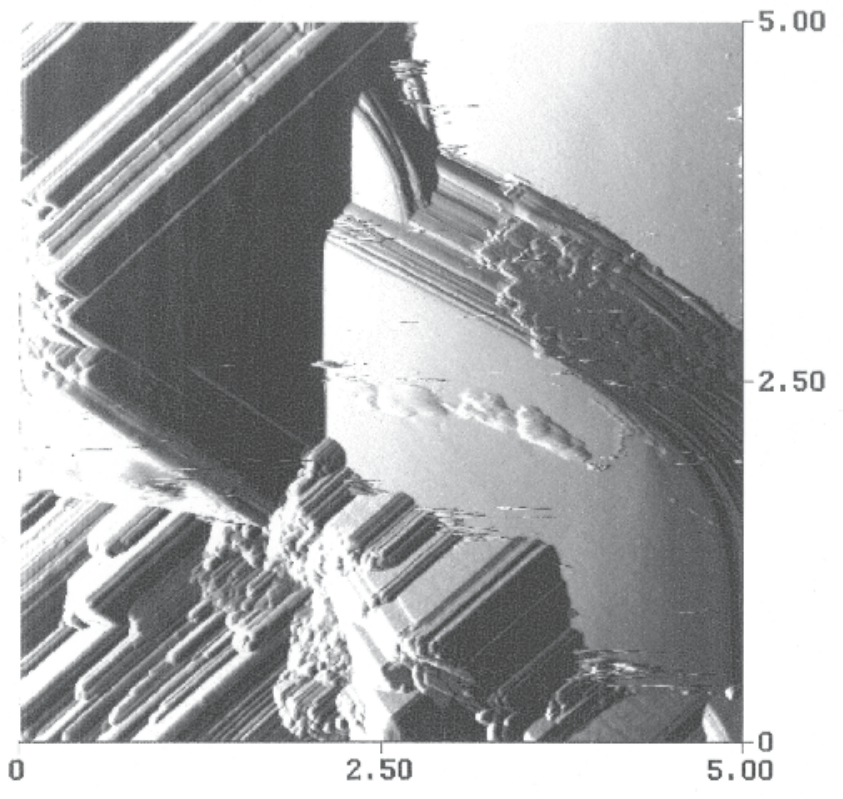

$\mu \mathrm{M}$

Fig. 5.- Imagen de deflexión AFM, obtenida para la membrana M03.

membrana, e es la porosidad, $\mathrm{rh}$ el radio de poro, $\Delta \mathrm{x}$ el espesor de la membrana y $\eta$ la viscosidad del agua. De esta forma, el radio hidráulico de poro se calcula como (4):

$$
\mathrm{r}_{\mathrm{h}}=\left(\frac{8 \mathrm{~L}_{\mathrm{p}} \Delta \mathrm{x} \eta}{\varepsilon}\right)^{\frac{1}{2}}
$$

Las porosidades se han determinado por diferencia de pesada de la membrana seca y la membrana empapada en agua. Los resultados de $\varepsilon$, junto con los radios obtenidos para el soporte y las membranas simétricas están recogidos en la Tabla III. En las membranas soportadas no es posible utilizar éste método pues se debería conocer la porosidad de cada una de las capas por separado y su espesor. En este caso, se debería partir de la relación que expresa la permeabilidad de una membrana formada por varias capas en función de la resistencia (7),

$$
\mathrm{L}_{\mathrm{p}}=\frac{1}{\mathrm{R}_{\mathrm{T}}}=\frac{1}{\mathrm{R}_{\mathrm{A}}+\mathrm{R}_{\mathrm{S}}}
$$

Donde $R_{T}$ es la resistencia total de la membrana, $R_{A}$ es la de la capa activa y $R_{S}$ es la del soporte. Teniendo en cuenta las ecuaciones anteriores,

$$
\mathrm{r}_{\mathrm{hA}}=\sqrt{\frac{\Delta \mathrm{x}_{\mathrm{A}}}{\varepsilon_{\mathrm{A}}\left(\frac{1}{8 \mathrm{~L}_{\mathrm{p}} \eta}-\frac{\Delta \mathrm{x}_{\mathrm{B}}}{\varepsilon_{\mathrm{B}} \mathrm{r}_{\mathrm{hB}}{ }^{2}}\right)}}
$$

Aunque el espesor de la capa selectiva se ha determinado por microscopía electrónica de barrido, obteniéndose un espesor entre 30 y $40 \mu \mathrm{m}$, y podría suponerse que la porosidad es igual a la de las membranas simétricas, el método no parece fiable. De hecho, si se realizan estos cálculos, los resultados obtenidos son incongruentes. Posiblemente, se debe a una gran diferencia entre porosidad de la membrana simétrica con

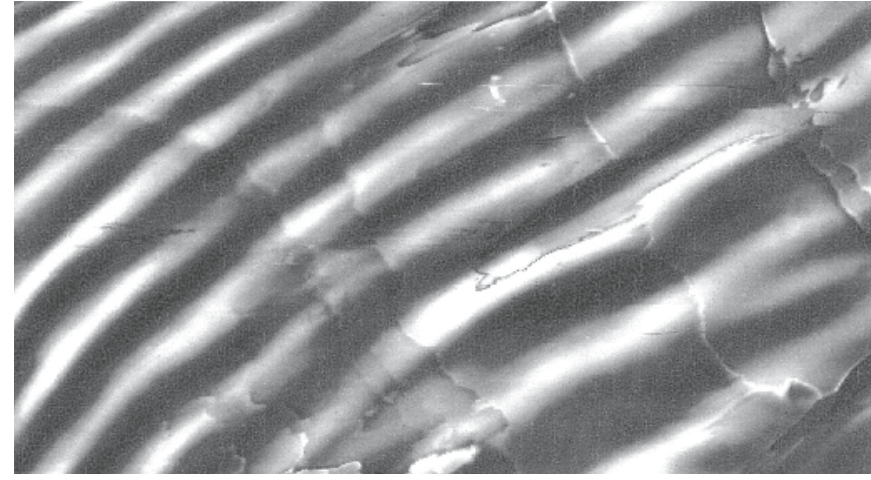

Fig. 6.- Imagen AFM de fricción, para la membrana M06.

la correspondiente soportada, debido a que el proceso de secado es muy diferente. En un caso, se realiza sobre moldes de yeso y en otro sobre el soporte poroso. Si se suponen válidos los datos de tamaño de poro obtenidos para las membranas simétricas, y se intenta calcular la porosidad, mediante la ecuación anterior, se obtiene: $15 \%, 4 \%, 1 \%$ y 3\% para la MS01, MS03, MS06 y MS09, respectivamente. Estos resultados son mucho más bajos de lo que cabría esperar. Posiblemente lo que ocurre en la realidad es que entre las membranas asimétricas y simétricas hay un cambio simultáneo de los dos parámetros, radio de poro y porosidad que explicaría este comportamiento aparentemente anormal. Teniendo en cuenta estos dos factores, junto con la posibilidad de una variabilidad en el espesor debido a las diferencias en las propiedades físicas de la pasta (viscosidad y densidad), hace pensar en la necesidad de otros métodos alternativos para una determinación más exacta del tamaño de poro como podrían ser: la porometría gaslíquido, la porosimetría de mercurio o la retención de solutos $(6,8)$. Estas posibilidades podrían ser objeto de futuras investigaciones.

\subsection{Topografía Superficial}

En la Figura 3 se muestra una imagen topográfica de la superficie de la membrana M01. Debido a la alta rugosidad de las muestras, en ningún caso se pudo determinar con una cierta fiabilidad mediante análisis de imagen la distribución de tamaños de poro ni la distribución de tamaños de partícula. No obstante, un análisis cualitativo de las imágenes, permite afirmar que las distribuciones de tamaño de partícula son similares a las obtenidas por rayos- $X$ para las pastas empleadas en su fabricación.

Un parámetro importante en la caracterización superficial de las membranas es la rugosidad, la cual está directamente relacionada con las características de ensuciamiento de la misma. Este parámetro se determina midiendo las alturas de cada punto de la superficie respecto al valor de referencia $Z$. Existen varias posibilidades de definir parámetros relacionados con la porosidad, pero uno de los más usados es la rugosidad media definida como (9):

$$
\mathrm{R}_{\mathrm{a}}=\frac{1}{\mathrm{n}} \sum_{\mathrm{i}=0}^{\mathrm{n}}\left|\mathrm{Z}_{\mathrm{i}}-\mathrm{Z}_{\mathrm{m}}\right|
$$

Donde $\mathrm{n}$ es el número total de puntos en la matriz de ima- 
gen, $Z_{\mathrm{i}}$ es la altura de cada punto y $\mathrm{Z}_{\mathrm{m}}$ es la altura media. Del análisis de la rugosidad de las membranas estudiadas, se obtiene que el mayor valor para este parámetro se encuentra en el soporte, seguido por las membranas soportadas y finalmente las simétricas son las menos rugosas. Evidentemente, dentro de cada grupo de membranas, la rugosidad aumenta con el tamaño de poro. El hecho de que la rugosidad de las membranas soportadas sea mayor que las simétricas debe estar relacionado con que la alta rugosidad del soporte se traduce a la capa activa debido a que ésta es delgada. Esto no ocurre con las fabricadas sobre moldes de yeso pues su espesor es de $1 \mathrm{~mm}$ y la rugosidad del soporte no afecta a la superficie. Como un ejemplo, en la Figura 4 se muestran dos imágenes tridimensionales para las membranas M01 y MS01 en las que se puede apreciar la diferencia de rugosidad.

Por otro lado, en las imágenes de AFM (ver Figuras 3 y 4) se observa que las partículas que forman la superficie de la membrana tienen estructura cristalina. Estos efectos pueden ser resaltados analizando imágenes obtenidas a partir de la deflexión del cantilever, como se muestra en la Figura 5 para la membrana M03. La configuración observada en esta figura, hace pensar que las características de dureza no serán homogéneas, sino que deben estar determinadas por las direcciones preferenciales de los cristales. Esto ha sido comprobado analizando imágenes en las que se analiza la fricción de la punta sobre la superficie, como se puede observar en la Figura 6 para un área de $4 \times 2 \mu \mathrm{m}^{2}$ de la membrana M06.

\section{CONCLUSIONES}

Como consecuencia de este trabajo, se pueden establecer una serie de conclusiones que se resumen en los siguientes enunciados:

a) Las membranas cerámicas para microfiltración que se han sintetizado poseen características de comportamiento similares a las membranas comerciales.

b) La posibilidad de la construcción de las membranas con distintas configuraciones, ayuda en gran medida a una mas completa caracterización de las membranas. c) Conforme al método de permeabilidad hidráulica se han determinado de los tamaños de poro de las membranas.

d) La utilización de Microscopía de fuerza Atómica (AFM) ha permitido obtener diversas características estructurales como: topografía, rugosidad, y fricción.

\section{AGRADECIMIENTOS}

Los autores argentinos agradecen el apoyo del Centro Regional de Estudios Avanzados Científicos y Tecnológicos (CREACyT) y del Laboratorio de Ciencias de Superficies y Medios Porosos (UNSL)- FONCyT . Los autores españoles agradecen la financiación de los siguientes proyectos de Investigación: QUI96-0767 del "Plan Nacional de Investigación y Desarrollo" (CICYT) y VA10496 de la Junta de Castilla y León.

\section{BIBLIOGRAFÍA}

1. M. Mulder, Basic Principles of Membrane Technology, Kluwer Acad. Pub. Dordrecht, The Netherlands, (1991).

2. R.R. Bhave, Inorganic Membranes: Synthesis, Characteristics and Applications, Van Nostrand Reinhold, New York, USA, (1991).

3. A. Hernández, P. Prádanos, J.I. Calvo, L. Palacio, Membranas cerámicas y su utilidad en procesos de separación, Bol. Soc. Cerám. Vidrio, 38 [3] 185-192 (1999).

4. K. Scott, Handbook of Industrial Membranes, Elsevier, Oxford, (1995).

5. L. Palacio, P. Prádanos, J.I. Calvo, A. Hernández, Caracterización de una nueva membrana cerámica de microfiltración con soporte de tejido en acero inoxidable flexible, Bol. Soc. Cerám. Vidrio, 38 [2] 117-120 (1999).

6. J. Marchese, Membranas: Procesos con Membranas, Editorial Universitaria de San Luis, San Luis, Argentina, (1995).

7. P. Prádanos, A. Hernández, J.I. Calvo y F. Tejerina, Mechanisms of Protein Fouling in Cross-flow UF through an Asymmetric Inorganic Membrane, J. Membr. Sci., 114, 115-126 (1996)

8. A. Hernández, J.I. Calvo, P. Prádanos y L. Palacio, A Multidisciplinary Approach Towards Pore Size Distributions of Microporous and Mesoporous Membranes, en Surface Chemistry and Electrochemistry of Membranes (T.S. Sorensen, ed.), Marcel Dekker Inc., New York, (1999)

9. P. Prádanos, M.L. Rodriguez, J.I. Calvo, A. Hernández, F. Tejerina and J.A. de Saja, Structural Characterization of an UF Membrane by Gas Adsorptiodesorption and AFM Measurements, J. Membr. Sci. 117 291-302 (1996).

Recibido: 21-10-98

Aceptado: 8-11-99 